\title{
Observational Constraints on Open Inflation Models
}

\author{
Martin White ${ }^{1}$ and Joseph Silk ${ }^{2}$ \\ ${ }^{1}$ Enrico Fermi Institute, University of Chicago \\ Chicago, IL 60637 \\ ${ }^{2}$ Center for Particle Astrophysics and Departments of Astronomy and Physics \\ University of California, Berkeley CA 94720
}

\begin{abstract}
We discuss observational constraints on models of open inflation. Current data from large-scale structure and the cosmic microwave background prefer models with blue spectra and/or $\Omega_{0} \geq 0.3-0.5$. Models with minimal anisotropy at large angles are strongly preferred.
\end{abstract}

In this Letter we examine the parameter space allowed by models of structure formation in a universe of subcritical density in which the main constituent is cold dark matter $(\mathrm{CDM}): \Omega_{\mathrm{CDM}} \equiv \Omega_{0}<1$. In spatially flat models with a cosmological constant, the initial fluctuation spectrum is well-defined and such models have received considerable attention [1,2]. However there is rather more structure in open inflationary models [3, [4, 5], and we believe it is timely to consider the constraints that arise from the formation of large-scale structure and the observed anisotropies in the cosmic microwave background (CMB). We apply these constraints to provide guidance for constructing open inflationary models by emphasizing the types of anisotropy spectra which are preferred by the data. None of the models currently discussed in the literature have the features necessary for a low- $\Omega_{0}$ universe with a Hubble constant $>60-65 \mathrm{~km} \mathrm{~s}^{-1} \mathrm{Mpc}^{-1}$.

The closest progenitor of this work is that of [6], who looked at the large-scale structure constraints for open models normalized to the COBE 2-year data. An in-depth treatment of current open models with scaleinvariant spectra below the curvature scale has recently appeared in [7], although their emphasis differs from the present one. No previous discussions have focussed on the full range of allowable parameter space in CDM models, including both large-scale structure and CMB constraints.

Both observational evidence and considerations of inflation [1,3, 4, motivate a model possessing adiabatic fluctuations and CDM, with, if the universe is indeed at subcritical density, preferably $\Omega_{0} \in[0.1,0.5]$. Direct measures of the Hubble constant appear to be approaching a consensus that $h=0.65 \pm 0.1$, where $H_{0}=$ $100 h \mathrm{~km} \mathrm{~s}^{-1} \mathrm{Mpc}^{-1}$. We will therefore focus attention on this range of $\left(\Omega_{0}, h\right)$ parameter space. All of our models will be normalized to the 4-year COBE-DMR data 8$]$ as described in [9]. Specifically, we take the amplitude of the density fluctuations at horizon-crossing to be

$$
\delta_{\mathrm{H}}\left(n, \Omega_{0}\right)=1.95 \times 10^{-5} \Omega_{0}^{-0.35-0.19 \ln \Omega_{0}-0.17 \tilde{n}}
$$

$$
\exp \left[-\widetilde{n}-0.14 \widetilde{n}^{2}\right]
$$

where $\widetilde{n}=n-1$ and $\delta_{\mathrm{H}}$ is related to the matter power spectrum today by

$$
\begin{aligned}
\Delta^{2}(k) & \equiv \frac{k^{3} P(k)}{2 \pi^{2}} \\
& \equiv\left(\frac{k}{H_{0}}\right)^{3+n} \delta_{\mathrm{H}}^{2} T^{2}(k),
\end{aligned}
$$

and $T(k)$ is the matter transfer function which depends on the cosmological parameters $\Omega_{0}$ and $h$. This normalization assumes that the fluctuations in the gravitational potential are a power law, $k^{n-1}$, in the eigenvalue $k$ of the Laplacian and that only sub-curvature scalar perturbations give rise to the $\mathrm{CMB}$ anisotropy. We will return to these points in detail later.

Restricting ourselves to open inflationary models, our parameter space consists of $\Omega_{0}<1, n, h$ and $\Omega_{\mathrm{B}} h^{2}$. There are a variety of observational tests which any model of structure formation must pass (see e.g. [10,6,2]). For our models, the most constraining tests, based on the well defined linear theory predictions, are the shape of the CDM power spectrum $\Delta^{2}$ [1] and the cluster abundance [12]. For the former, we perform a $\chi^{2}$ fit directly to the data for $\Delta^{2}$ as tabulated in [11], excluding the last 4 points and allowing the overall normalization to float. The latter constraint can be expressed as a limit on

$$
\sigma_{8}^{2}=\int \frac{\mathrm{d} k}{k} \Delta^{2}(k) W^{2}(k R)
$$

where $R=8 h^{-1} \mathrm{Mpc}$, which probes scales $k \sim$ $0.2 h \mathrm{Mpc}^{-1}$. The amount of small-scale power required to fit the cluster abundance in these models is [12],

$$
\sigma_{8}=0.6 \Omega_{0}^{-0.36-0.31 \Omega_{0}+0.28 \Omega_{0}^{2}}
$$

where the errors are $+32 \% \times \Omega_{0}^{0.17 \log _{10} \Omega_{0}}$ and $-24 \%$ $\times \Omega_{0}^{0.17 \log _{10} \Omega_{0}}$ at $95 \% \mathrm{CL}$. To this we add in quadrature a $2 \sigma$ error from the $C O B E$ normalization of $20 \%$. We have 


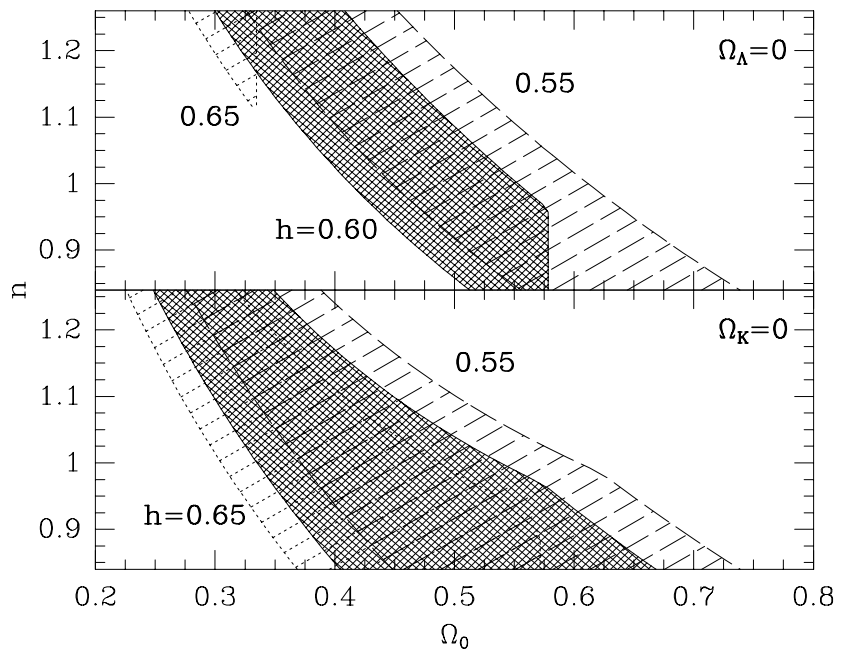

FIG. 1. Allowed regions: The values of $\Omega_{0}$ and $n$, for fixed $h$, for which CDM models do not violate any of our large-scale structure constraints at $95 \% \mathrm{CL}$. Upper limits on $\Omega_{0}$ come from requiring $t_{0} \geq 12 \mathrm{Gyr}$, other constraints are discussed in the text. We have assumed $\Omega_{\mathrm{B}} h^{2}=0.015$ and minimal large-angle $\mathrm{CMB}$ anisotropies.

also checked that the abundances of high redshift massive objects (e.g. quasars and damped Ly $\alpha$ systems) are compatible with the observations for all of these models. Further, we require that our models are at least $12 \mathrm{Gyr}$ old [13], which provides an upper limit to the value of $\Omega_{0}$ which we can consider for any fixed Hubble constant. Since we will be concerned here mostly with the lower limits to $\Omega_{0}$, the precise age constraint that we impose will not be important.

We show in Fig. 1 the region of parameter space allowed after applying these constraints for a variety of slices with $h=$ constant. We have fixed the baryon content of the universe at this stage to $\Omega_{\mathrm{B}} h^{2}=0.015$. Our conclusions do not depend strongly on this assumption for the range of $\Omega_{0}$ we consider. Lowering $\Omega_{\mathrm{B}} h^{2}$ allows slightly higher $\Omega_{0}$ for fixed $h$ while increasing it allows slightly lower $\Omega_{0}$.

Let us concentrate on the open models (top panel); we present the flat models (lower panel) merely for comparison. Notice that very low- $\Omega_{0}$ models are only allowed if the primordial fluctuation spectrum is "blue" $(n>1)$. Such blue spectra arise in "flat" inflation [14] mostly in hybrid or two-field models where inflation ends not by a field rolling down an ever steepening potential but through an instability. To the best of our knowledge no models of open inflation have been constructed with this property, though models with "blue" specta may be more natural in open inflation 四.

The problem that arises in fitting the data to these models is due to the low $C O B E$ normalization 15,9 . In each case the upper limit on $\Omega_{0}$ for fixed $n$ comes from the shape of the CDM power spectrum, while the lower limit comes from the cluster abundance. This latter constraint scales roughly as $\Omega^{-0.4}$ while the COBE normalization at large scales is almost flat with $\Omega_{0}$. At fixed $h$, lowering $\Omega_{0}$ changes the shape of the CDM transfer function so as to reduce small-scale power. The lowest $\Omega_{0}$ allowed occurs for the largest ratio of small- to large-scale power, i.e. when we tilt the primoridal power spectrum to $n>1$ or increase the Hubble constant. Less tilt, or lower $\Omega_{0}$, could be accomodated if $\sigma_{8}$ inferred from the cluster abundances were lowered. However we have already adopted conservative uncertainties on this quantity.

In producing Fig. 1, we have normalized the models to the COBE data assuming that the primordial spectrum of curvature perturbations is a power-law in the eigenvalue of the Laplacian. Ignoring a possible "running" of the spectral index, this is reasonable on scales much smaller than the curvature scale. However open inflation models can predict departures from simple powerlaw behaviour near the curvature scale. These departures only affect the lowest multipoles of the CMB anisotropy spectrum, and hence the $C O B E$ normalization. For current models, departures from a power-law on $C O B E$ scales do not affect the normalization significantly [16], although models that decreased the anisotropy at fixed $\delta_{\mathrm{H}}$ would provide a better fit to the data. More importantly, we have not included any contribution from "super-curvature modes" [17], "bubble wall modes" 18] or tensor anisotropies. Since the COBE measurement of the temperature anisotropies is fixed, any of these "extra" contributions to the anisotropy would lower $\delta_{\mathrm{H}}$, worsening the agreement with observations. For example, decreasing $\delta_{\mathrm{H}}$ by a factor of 1.5 reduces the allowed region to a thin band, $\sim 1 / 5$ of the width, along the upper right of the regions shown in Fig. 1. Reducing $\delta_{\mathrm{H}}$ by a factor of 2 causes the allowed regions shown in Fig. 1 to disappear entirely (a small region persists at high $\Omega_{0}$ and $n \sim 0.7-0.8$, which is off our plot).

Thus the challenge to model builders interested in constructing open universe inflationary models is to provide "blue" spectra with a minimal amount of largeangle CMB anisotropy. If we imagine that obtaining very "blue" spectra $(n \geq 1.1-1.2)$ is as difficult in the open models as it is in the flat models (see e.g. [14]), then open models will require a relatively low Hubble constant and a relatively high density: $\Omega_{0} \geq 0.4-0.5$. If the models are significantly "blue", then they will not look like the usual scale-invariant CDM models parameterized by $\Gamma \simeq \Omega_{0} h$. Also should the models have power-law spectra which stay "blue" to very small scales, limits from the production of spectral distortions [19] and primordial black holes [20] need to be considered.

Another strong constraint on open models comes from small angular scale measurements of the CMB anisotropy. It has long been realized 21,22,23] that the observation of any feature in the $\mathrm{CMB}$ angular power 


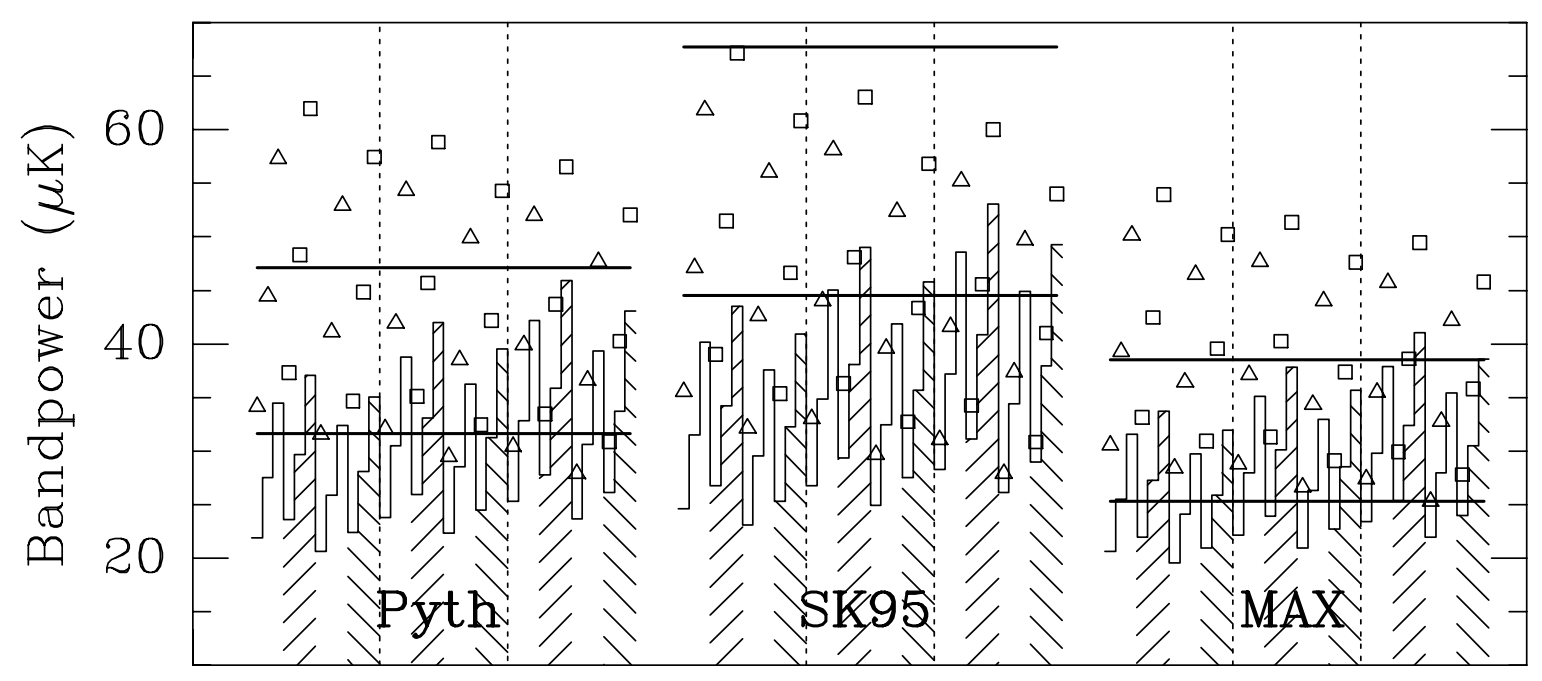

FIG. 2. CMB predictions: The bandpower predicted, as a function of "model number", for the Python, Saskatoon and MAX experiments, with 95\%CL measurements (solid, horizontal lines) excluding calibration uncertainty (Py: 20\%, SK: 14\%, MAX: $10 \%$ ) and $10 \%$ COBE normalization uncertainty. The histogram represents open models. Unshaded models have $\Omega_{\mathrm{B}} h^{2}=0.01$, shaded models have $\Omega_{\mathrm{B}} h^{2}=0.02$. Within each group $n=0.8,1,1.2$. Shading running bottom-left to top-right indicates $h=0.6$, top-left to bottom-right $h=0.7$, with the unshaded predictions having the same $h$ as the shaded prediction to their right. Vertical dotted lines separate $\Omega_{0}=0.3,0.4$ and 0.5 (left to right). The open triangles and squares are the predictions for $\Omega_{\Lambda}=1-\Omega_{0}$ for comparison.

spectrum would allow one to perform the classical "angular diameter distance" cosmological test of the (spatial) curvature of the universe (e.g. 24]). In an open universe, the peaks in the angular power spectrum of a CDM model shift to smaller angular scale. Thus experiments which probe angular scales around $0.5^{\circ}$ will observe more power in a model without spatial curvature than with negative spatial curvature. To quantify this we have chosen 3 representative experiments which probe scales near the first peak in a flat model: Python [25], Saskatoon [26] and MAX [27]. For Python we have taken the highest $\ell$ bandpower, for Saskatoon the 3rd of 5 bandpowers and for MAX the "combined" analysis. We show in Fig. 2 the predicted bandpower (also known as $Q_{\text {flat }}$, see [28]), or level of fluctuation, for 32 models with $\Omega_{0}=0.3,0.4$, 0.5 and $n=0.8,1.0,1.2$. Since the CMB anisotropies are sensitive to $h$ and $\Omega_{\mathrm{B}} h^{2}$ we have computed them for $h=0.6,0.7$ and $\Omega_{\mathrm{B}} h^{2}=0.01,0.02$. Interpolation to other values near these is stable. These predictions were computed by numerical evolution of the coupled Einstein, Boltzmann and fluid equations as discussed in 29, using the window functions provided by the authors of 25,26, 27. We have not included late reionization, which would lower the predictions by $\exp (-\tau)$ where $\tau$ is the optical depth to Thomson scattering between the redshift of reionization and today. This redshift is very uncertain, our best guess (see e.g. [30]) puts $\tau$ in the range $\sim 1 \%$ to
$30 \%$.

The trends in Fig. 2 are easy to understand. Higher $n$ means more fluctuation power at $0.5^{\circ}$ since the models are normalized to $C O B E$ on large scales. Similarly, higher $\Omega_{\mathrm{B}} h^{2}$ means more power on small scales since it enhances the amplitude of the acoustic oscillations in the baryon-photon plasma before recombination. Lowering $h$ shifts matter-radiation equality closer to last-scattering, thus enhancing the acoustic oscillations due to the decay of the potentials. For the open models, lowering $\Omega_{0}$ enhances the peak height (due to the shift of equality), but it also shifts the peak to smaller angular scales (out of the experimental window) and increases the anisotropy at large-scales (which affects the COBE normalization). The net effect is to lower the predicted power at $0.5^{\circ}$. For the $\Omega_{\Lambda}$ models the enhanced peak height serves to raise the predicted power at $0.5^{\circ}$.

Providing enough power at small (now angular) scales is confirmed to be a problem for open models with $\Omega_{0} \ll 1$. We conclude that those models best able to fit the data have $n>1, \Omega_{0} \approx 0.5$, a relatively low Hubble constant and a high $\Omega_{\mathrm{B}} h^{2}$. Any "extra" contribution to the large-angle anisotropy over what we have assumed, such as super-curvature modes, bubble wall fluctuations or gravitational waves, would worsen the situation.

In summary, we have compared models of open inflation with the available data on the shape of the CDM 
power spectrum, the abundance of rich clusters and small scale CMB anisotropies. The COBE-DMR normalized models prefer a relatively high $\Omega_{0} \geq 0.3$ and/or "blue" spectra $n \geq 1$. Models in which "extra" CMB anisotropies are present are very strongly constrained: enough extra anisotropy to lower $\delta_{\mathrm{H}}$ by a factor of 2 leaves no allowed region on Fig. 1. The constraints become stropger should the universe contain some hot as well as cold dark matter, which reduces the amount of smallscale power (e.g. 31]). A similar reduction in small-scale power would be obtained by a large increase in $\Omega_{\mathrm{B}} h^{2}$ (e.g. [32]), especially at low $\Omega_{0}$. Alternatively if the $\sigma_{8}$ inferred from cluster abundance measures is lowered then the allowed region would be increased.

We acknowledge useful conversations with Joanne Cohn and Andrew Liddle.

[1] D. Scott, J. Silk, M. White, Science, 268, 829 (1995), astro-ph/9505015; L.M. Krauss, M.S. Turner, Gen. Rel. \& Grav., 27, 1137 (1995), astro-ph/9504003; J.P. Ostriker, P.J. Steinhardt, Nature, 377, 600 (1995), astro-ph/9505066

[2] A.R. Liddle, D.H. Lyth. P.T.P. Viana, M. White, MNRAS, 282, 281 (1996), astro-ph/9512102

[3] D.H. Lyth, E.D. Stewart, Phys. Lett. B252, 336 (1990); M. Sasaki, T. Tanaka, K. Yamamoto, J. Yokoyama, Phys. Lett. B317 510 (1993); B. Ratra, P.J.E. Peebles, Astrophys. J., 432, L5 (1994); M. Bucher, A.S. Goldhaber, N. Turok, Phys. Rev. D52, 3314 (1995), hep-ph/9411206; A.D. Linde, Phys. Lett. B351, 99 (1995), hep-ph/9503097; A.D. Linde, A. Mezhlumian, Phys. Rev. D52, 6789 (1995), astro-ph/9506017; A.M. Green, A.R. Liddle, Phys. Rev. D, submitted, astro-ph/9607166

[4] M. Bucher, N. Turok, Phys. Rev. D52, 5538 (1995), hep$\mathrm{ph} / 9503393$

[5] J.D. Cohn, in Proceedings of the XXXIth Rencontres de Moriond, Microwave Background Anisotropies, astro$\mathrm{ph} / 9606052$

[6] A.R. Liddle, D.H. Lyth, D. Roberts, P.T.P. Viana, MNRAS, 278, 644 (1996), astro-ph/9506091.

[7] K. Gorski, B. Ratra, R. Stompor, N. Sugiyama, A.J. Banday, astro-ph/9608054

[8] C.L. Bennett, et al., Astrophys. J., 454, L1 (1996), astro$\mathrm{ph} / 9601067$

9] EF. Bunn, M. White, A I., Jum ph/9607060

[10] M. White, P.T.P. Viana, A.R. Liddle, D. Scott, MNRAS, in press, astro-ph/9605057; A.R. Liddle, D.H. Lyth, R.K. Schaefer, Q. Shafi, P.T.P. Viana, MNRAS, 281, 531 (1996), astro-ph/9511057;

[11] J.A. Peacock, S.J. Dodds, MNRAS, 267, 1020 (1994), astro-ph/9311057.

[12] P.T.P. Viana, A.R. Liddle, MNRAS, 281, 323 (1996), astro$\mathrm{ph} / 9511007$
[13] B. Chaboyer, P.J. Kernan, L.M. Krauss, P. Demarque, astro-ph/9509115.

[14] A.R. Liddle, D.H. Lyth, MNRAS, 265, 379 (1993); A. Linde, Phys. Rev., D49, 748 (1994); S. Mollerach, S. Matarrese, F. Lucchin, Phys. Rev., D50, 4835 (1994), astro-ph/9309054; E.J. Copeland, A.R. Liddle, D.H. Lyth, E.D. Stewart, D. Wands, Phys. Rev., D49, 6410 (1994), astro-ph/9401011; J. Garcia-Bellido, D. Wands, astro$\mathrm{ph} / 9606047$

[15] M. White, D. Scott, Comments Astrophys., 18, 289 (1996), astro-ph/9601170.

[16] K. Yamamoto, E.F. Bunn, Astrophys. J., 464, 8 (1996), astro-ph/9508090

[17] M. Sasaki, T. Tanaka, K. Yamamoto, Phys. Rev. D51, 2979 (1995); K. Yamamoto, E.F. Bunn, astro-ph/9508090; M. Sasaki, T. Tanaka, astro-ph/9605104.

[18] A. Linde, A. Mezhlumian, Phys. Rev. D52, 6789 (1995), astro-ph/9506017; T. Hamazaki, M. Sasaki, T. Tanaka, K. Yamamoto, Phys. Rev. D53 2045 (1996); J. Garriga, grqc/9602025; J. Garcia-Bellido, astro-ph/9510029.

[19] W. Hu, D. Scott, J. Silk, Astrophys. J., 430, L5 (1994), astro-ph/9402045.

[20] B.J. Carr, J.H. Gilbert, J.E. Lidsey, Phys. Rev. D50, 4853 (1994), astro-ph/9405027.

[21] A.G. Doroshkevich, YaB Zel'dovich, R.A. Sunyaev, Sov. Astron., 22, 523 (1978).

[22] M.S. Wilson and J. Silk, Astrophys. J., 243, 14 (1981).

[23] N. Sugiyama, N. Gouda, Prog. Theor. Phys., 88, 803 (1992); M. Kamionkowski, D. Spergel, N. Sugiyama, Astrophys. J., 426, L57 (1994), astro-ph/9401003.

[24] P.J.E. Peebles, "Principles of Physical Cosmology", (Princeton, NJ) 1993.

[25] S.R. Platt, J. Kovac, M. Dragovan, J.B. Peterson, J.E. Ruhl, Astrophys. J., in press, astro-ph/9606175

[26] C.B. Netterfield, M.J. Devlin, N. Jarosik, L. Page, E.J. Wollack, Astrophys. J., in press, astro-ph/9601197

[27] S. Tanaka, et al., in preparation (1996).

[28] M. White, D. Scott, in CMB Anisotropies 2 Years After COBE: Observations, Theory and the Future, ed. L. Krauss, p. 254, astro-ph/9406060.

[29] W. Hu, D. Scott, N. Sugiyama, M. White, Phys. Rev. D52, 5498 (1995), astro-ph/9505043.

[30] H.M.P. Couchman, MNRAS 214, 137 (1985). H.M.P. Couchman, M.J. Rees, MNRAS 221, 53 (1986); M. Fukugita, M. Kawasaki, MNRAS 269, 563 (1994); S. Sasaki, F. Takahara, Y. Suto, Prog. Theor. Phys. 90, 85 (1993); M. Tegmark, J. Silk, Blanchard, Astrophys. J. 420, 484 (1993) (erratum 434, 395); A. Liddle, D.H. Lyth, MNRAS 273, 1177 (1995), astro-ph/9409077

[31] J. Primack et al., Phys. Rev. Lett., 74, 2160 (1995)

[32] S. Burles, D. Tytler, submitted to Science, astro$\mathrm{ph} / 9603070$ 